\title{
Crisis de los cuidados y servicios sociales
}

\section{Fernando Fantova}

Consultor social

$<$ fernando@fantova.net>
Artikulu honetan aztergai hartzen da -izaera kritiko eta proposizio moduan - zainketen inguruan gizarte-zerbitzuek betetzen duten rola, berariaz zainketen krisialdi gisara, gehienbat ekonomia feministak izendatu duen testuinguruaren baitan (bereziki nabarmenduz Espainiako egoera). Lehenik, aztertzen dira zainketen kontzeptua eta zainketen erlazio primarioak. Horren ondoren, aztertzen dira eskuartze profesionalen eta erlazio primarioen kontzeptua, betiere, erreferentzia gisara politika publikoak eta arlo horretan jarduten duten sektoreak kontuan hartuz. Horretaz gain, interpretazio bat burutzen da zainketen krisialdiaren eta politika sozialen inguruan, horren ostean, interakzioa babestu eta sustatzen duten zerbitzu gisara (autonomia funtzionala eta erlazioen integrazioa) proposaturiko gizarte-zerbitzuen arloa aztertzera igarotzeko. Bukaeran, artikuluak proposatzen du zainketen krisialdiari gizarte-zerbitzuek eta Ongizate Estatuak erantzun ahal izateko agenda estrategiko bat, eta kontrasterako erreferentzia gisara aurkezten da Erresuma Batuko gizarte-zerbitzuen inguruko eztabaida.

\section{GAKO-HITZAK:}

Zainketa primarioak, zainketa profesionalak, zaintzaren krisialdia, gizarte-zerbitzuak, politika sozialak.
El artículo analiza -en clave crítica y propositivael papel de los servicios sociales en lo relacionado con los cuidados, específicamente en el contexto de la que se ha dado en llamar -por parte, fundamentalmente, de la economía feministacrisis de los cuidados (con especial referencia a España). Se aborda, inicialmente, el concepto de cuidado y las relaciones primarias como relaciones -también- de cuidado. Posteriormente se conceptualiza y estudia la interrelación entre intervención profesional y relaciones primarias, con referencia a las políticas públicas y sectores de actividad implicados. A continuación, se interpreta la crisis de los cuidados y la respuesta de las políticas sociales, para pasar a centrarse en el ámbito de los servicios sociales, propuestos como servicios que protegen y promueven la interacción (autonomía funcional e integración relacional). El artículo termina proponiendo una agenda estratégica para una respuesta de los servicios sociales y el sistema de bienestar a la crisis de los cuidados y ofreciendo una referencia de contraste: el debate sobre servicios sociales en el Reino Unido.

\section{Palabras Clave:}

Cuidados primarios, cuidados profesionales, crisis de los cuidados, organización social de los cuidados, servicios sociales, políticas sociales. 
Las organizaciones antagonistas trataron de ir más lejos, cuestionando los sometimientos característicos de las estructuras comunitarias arcaicas y tratando de filtrar aquellas sedimentaciones opresoras. Seguramente fue un paso en falso. No porque fuera una mala idea, sino porque intentaron hacerlo rompiendo con la ética del cuidado y la codependencia a través del objetivismo. Tal vez por eso los revolucionarios han estado mucho más interesados por las falsas promesas de las ciencias sociales que por la

ética. Y tal vez por eso la burocratización de los cuidados mutuos a través de un sistema racional e impersonal ha sido una de las grandes amenazas a las que se han enfrentado los proyectos de emancipación. No sé si el izquierdismo es la enfermedad infantil del comunismo, pero desde luego la burocracia es su demencia senil (Rendueles, 2013: 152)

\section{1. ¿Por qué este artículo?}

Este artículo nace de la necesidad sentida por el autor de intentar hacer una contribución en lo tocante al establecimiento -clarificación, fundamentación, articulación, ajuste- de algunos términos de referencia utilizados en el estudio y debate sobre la organización social de los cuidados - particularmente- en España (aunque sin renunciar a que las aportaciones puedan resultar significativas y útiles en contextos más amplios), de modo que dicho estudio y debate puedan ser más productivos a corto plazo para la toma de decisiones en políticas sociales. Se intentará recorrer un itinerario lógico en el que se responda a las siguientes preguntas:

- ¿De qué hablamos, exactamente, cuando hablamos de cuidados?

- ¿En qué medida y en qué sentido cabe hablar de cuidados profesionales?

- ¿Pueden considerarse los cuidados profesionales como una prestación característica de alguno de los sectores de actividad pertenecientes al ámbito de la acción pro bienestar?

- ¿De qué hablamos cuando hablamos de crisis de los cuidados?

- ¿En qué medida y en qué sentido son o pueden ser los servicios sociales un elemento clave en la organización social de los cuidados en España?

- ¿Cuáles serían las - o al menos algunascondiciones de posibilidad para una contribución más significativa de los servicios sociales a la respuesta a la crisis de los cuidados (considerando el debate en el Reino Unido?

La escritura del artículo se realiza desde la percepción -en la que no cabe detenerse aquíde una cierta confusión en las formulaciones -tanto políticas y legislativas como académicas y técnicas- y de una cierta fragmentación e incomunicación entre diversas comunidades de conocimiento o de práctica relacionadas con el asunto que nos ocupa. El artículo intentará hacer aportaciones nuevas, aunque inevitablemente se apoyará en trabajos anteriores del autor; se nutrirá especialmente de aportaciones tomadas de la economía feminista - fundamentalmente a través de autoras españolas- y del debate sobre servicios sociales (y atención integrada) que tiene lugar en el Reino Unido. El autor agradece las sugerencias de mejora realizadas desde la coordinación del número de la revista en el que se incluye el artículo.

\section{2. ¿De qué hablamos, exactamente, cuando hablamos de cuidados?}

En buena medida, según señala Constanza Tobío, "ha sido la confluencia de la generalización de la actividad laboral femenina, incluyendo a las madres de niños, y de la reflexión feminista acerca de la importancia económica y social del trabajo realizado por las mujeres en el ámbito doméstico lo que ha despertado la conciencia y la preocupación acerca de la problemática del cuidado de las personas. Se habla de déficit en esta materia porque en el horizonte aparecen nuevas necesidades ligadas al envejecimiento demográfico, pero también porque se entiende que generan un derecho a ser atendidas del que responde la sociedad y porque las formas tradicionales de cuidar a las personas, en la familia y a cargo de las mujeres, no son ya posibles, ni se consideran deseables" (Tobío et al., 2010: 12).

En el contexto escogido en este artículo, el elemento clave que permite delimitar y distinguir a qué nos referimos cuando hablamos de cuidados es el hecho de que se trata de actividades que, en términos generales o mayoritarios, las personas pueden hacer por y para sí mismas. Denominaríamos cuidados a actividades que responden a necesidades básicas o fundamentales de las personas y que por su limitado grado de complejidad pueden ser - normal o habitualmente- realizadas por las propias personas para sí mismas. Es decir, se propone denominar cuidado a aquello que -en la mayor parte de los casos - sería autocuidado. Se trataría de "tareas muy variadas encaminadas a asegurar la nutrición, la higiene, el abrigo o el descanso" (ibídem). Mary Daly, al referirse al cuidado como bien a ser producido y como objeto a ser estudiado en el ámbito de las políticas sociales, señala que se trata del cuidado que reciben las personas que no pueden cuidarse a sí mismas (2002: 252).

Dentro de las áreas de conocimiento relevantes al respecto la referencia canónica, codificada o consensuada (también en la ley española sobre autonomía y dependencia) sería la de las denominadas actividades básicas de la vida diaria. Las ABVD se han definido como "operaciones mecánicas que realizan las personas en su ámbito doméstico y que les permiten dar respuesta a 
sus necesidades más primarias” especificándose que "dentro de las actividades básicas de la vida diaria cabría considerar la realización de cambios en las posiciones y ubicaciones del cuerpo y su mantenimiento; asearse y cuidar el propio aspecto; control de esfínteres y uso del servicio; vestirse, desvestirse y arreglarse; comer y beber [...]. A la hora de decir si una persona es capaz de realizar las actividades básicas de la vida diaria, aparte de la capacidad para su pura ejecución física, se toma en cuenta la capacidad cognitiva de tomar adecuadamente las iniciativas y decisiones correspondientes" (Fantova, 2010: 19).

Se propone, por tanto, entender el autocuidado como la realización - por y para la persona-de las actividades básicas de la vida diaria, sin que sea el momento para discutir si cabe incorporar aquí otras actividades no básicas - como las denominadas instrumentales - de la vida diaria. El cuidado por parte de otra persona sería, entonces, la asistencia suplementaria o complementaria en (o para) la realización de dichas actividades. Ello no quiere decir que la persona que cuida sólo brinde esa ayuda, sino que en este artículo se propone dicha ayuda como la que permite definir operativamente el concepto de cuidado. Parece evidente que, en la vida de las personas, lo más frecuente es que vivamos en circunstancias en las que -en mayor 0 menor medida- nos autocuidamos y en las que - simultáneamente- recibimos ayuda en algún grado, siendo ciertamente difícil de identificar situaciones de total autocuidado o de total cuidado por parte de otras personas.

Así pues, del mismo modo que se afirma que estas actividades que -en el contexto en el que se escribe este artículo- se denominan cuidados son, por definición, realizadas por las propias personas interesadas en la mayoría de los casos, se ha de afirmar también que todas las personas, en determinados momentos, épocas, circunstancias o situaciones de nuestra vida necesitamos que otras personas nos cuiden, que otras personas nos proporcionen esos que se denominan -aquícuidados. Así, recordará Cristina Carrasco que los cuidados "no solo se requieren en las primeras etapas de la vida, sino a lo largo de todo ciclo vital, aunque con especial intensidad en los inicios y finales del ciclo vital. De ahí que el cuidado sea universal (todos y todas lo requerimos) e inevitable (es absolutamente necesario para el desarrollo de la vida)" (2015: 52-53).

Lógicamente hay que ser consciente de que en la literatura científica, técnica, política o normativa sobre los cuidados cabe encontrar amplitudes y connotaciones diferentes a las que se proponen aquí y que, en todo caso, la expresión cuidado (en inglés care) es utilizada en sentidos diversos. Específicamente, cuando se habla de cuidados de larga duración (long term care), no se está pensando normalmente en los cuidados que necesitamos todas las personas en los primeros años de nuestra vida. En el contexto de este artículo, como se verá, se percibe o subraya más bien la equivalencia o continuidad de los cuidados en los diferentes momentos o circunstancias de nuestra vida y, por tanto, la continuidad o equivalencia entre los llamados cuidados de larga duración y el resto de los cuidados.

\section{3. ¿En qué medida y en qué sentido cabe hablar de cuidados profesionales?}

Para responder a esta pregunta se ha de plantear primero un esquema conceptual que ha resultado central en anteriores contribuciones (Fantova, 2014: 44-53). Según este esquema, las actividades, prestaciones, apoyos o, en general, aportaciones valiosas que las personas recibimos para dar respuesta a nuestras necesidades (aportaciones denominadas, en sentido amplio, bienes) tienen 0 adquieren diferente naturaleza o características en función del agente (o la esfera en) que nos proporcione dicha respuesta o bien y de la correspondiente lógica con la que se produzca o realice dicha respuesta a la necesidad, entrega del bien o aportación de valor, siendo que, como señala Rafael Aliena, no existe equivalencia funcional entre estas esferas (2012: 12), es decir, sus efectos pueden ser más o menos parecidos pero no iguales. El esquema, en el que no es posible detenerse aquí, se resume en el Cuadro 1.

\begin{tabular}{|l|l|l|}
\hline \multicolumn{3}{|l|}{ Cuadro 1. Esferas, bienes y lógicas en la esfera social } \\
\hline Agente & Bienes & Lógica \\
\hline Comunidad & Relacionales & Reciprocidad \\
\hline Estado & Públicos & Derecho \\
\hline Mercado & Privados & Intercambio \\
\hline Iniciativa social & Comunes & Solidaridad \\
\hline
\end{tabular}

Fuente: Elaboración propia.

Se entiende que los bienes tienen una cierta elasticidad - unos más y otros menos- a la hora de ser tratados como relacionales, públicos, privados o comunes, pero, también, que hay ciertas características intrínsecas de los diversos bienes que hacen que - unos más y otros menos- sean vistos y tratados como bienes de un tipo u otro. Así, por poner un ejemplo, las calles de las ciudades, que nos sirven para desplazarnos de un lugar a otro, tienen unas características en función de las cuales tienden a ser vistas y tratadas en nuestro contexto como bienes públicos, es decir, como bienes que todas las personas tenemos derecho a usar y de cuya gestión se encarga el Estado (sin que proceda ahora entrar en mayores precisiones o matices).

Pues bien, en lo que toca a los cuidados, cabría afirmar que tienen características que invitarían a verlos y tratarlos, en primera instancia, como bienes relacionales, es decir, como bienes que 
coproducimos y compartimos en las relaciones o vínculos primarios de carácter familiar y, en general, comunitario. Así, Cristina Carrasco, al estudiar el "cuidado como bien relacional" (2015: 52-54) apunta que "es curioso - o no, ya que la mirada masculina nunca se dirige al espacio doméstico- que el cuidado no se categorice habitualmente como bien relacional, teniendo en cuenta que precisamente ha sido el desarrollo de las relaciones mercantiles el que ha eliminado de las relaciones humanas lo que era y es la característica básica del cuidado: su dimensión relacional" (ibídem: 53).

Según Carrasco, "la identificación del cuidado como bien relacional es absolutamente directa: se trata de una relación-interacción entre personas concretas; existe una reciprocidad compartida libre de asumir o no, lo cual lo dota de una gran fragilidad (de hecho, el tema de los cuidados es el gran temor de las personas mayores); solo tiene lugar durante la relación; requiere de una historia temporal, la relación se va construyendo a través del cuidado; es de gran valor social, tanto que la vida y gran parte del bienestar depende de los cuidados; no puede asignarse un precio [...], la relación - sin negar que pueda existir- no es igual si el cuidado se realiza bajo relaciones capitalistas o bajo relaciones familiares" (ibídem).

La situación (que se entiende como) universal y que cabe tomar como referencia arquetípica sería la del cuidado de la criatura recién nacida. Cuando los seres humanos traen a otro al mundo se genera con él un vínculo que, incluso antes del propio nacimiento, entraña responsabilidades familiares y -en su seno- una relación de cuidado en el sentido establecido más arriba. Es tal la vulnerabilidad y dependencia de la criatura y tal el cuidado que quienes la han concebido sienten, normalmente, que deben proporcionarle, que cabe identificar un universal antropológico respecto a que ese vínculo y esa relación se producen en clave, fundamentalmente, de gratuidad - de don-es decir, que los progenitores brindan ese apoyo 0 ayuda -que, al menos inicialmente, es en buena medida cuidado - libremente, sin estar obligados externamente, sin reclamar nada a cambio y con una cierta expectativa de reciprocidad. Esa relación de cuidado, en condiciones normales, está atravesada de emociones, sentimientos o afectos fundamentales para el desarrollo humano que solemos resumir y expresar hablando de apego, confianza y, en definitiva, amor.

Es evidente que puede darse la circunstancia de que una criatura, incluso recién nacida, no tenga a su lado nadie -o nadie idóneo- con vínculos primarios (familiares o, en general, comunitarios) que la cuide y que, en ese caso, por ejemplo, identificamos más claramente la naturaleza pública del cuidado y la responsabilidad pública al respecto, responsabilidad que, en cualquier caso, existe y ha de ser ejercida. Pero también es evidente que es preferible la situación en la que el cuidado es asumido o construido, en primera instancia, como bien relacional y en que la criatura dispone de personas idóneas dispuestas para su cuidado que estén y se sientan primariamente vinculadas a ella. Aunque el sentido común lo señala con suficiente claridad, la evidencia científica al respecto es abundante (Casado y Sanz, 2012: 5-8).

Es, obviamente, discutible y discutido - de manera diferente en diversos contextos culturales y desde distintas visiones morales - en qué medida y en qué sentido esta identificación y caracterización de la dimensión relacional del cuidado que se ha planteado para el caso de la criatura recién nacida es trasladable a otras situaciones o momentos vitales en los que las personas necesitamos cuidados. Podría decirse que sí, en tanto en cuanto más básicas fueran las actividades en cuestión y en tanto en cuanto mayor fuera la vulnerabilidad de la persona, dado que en esa misma medida parecen más indicadas las connotaciones de donación, intimidad, proximidad o confianza que se esperan de las relaciones primarias. Serge Guérin, por cierto, recuerda que, dentro de las personas cuidadoras no profesionales (unos 3,5 millones en Francia), un $18 \%$ no tiene vínculos biológicos o civiles con las personas a las que cuidan sino que son vecinas, amigas o colegas (2010:107).

Se estaría intentando, en todo caso, identificar la esencia o naturaleza de las relaciones primarias, dibujar su valor civilizatorio, caracterizar esos bienes relacionales que coproducimos en la enorme diversidad de modelos familiares y comunitarios y en la propia diversidad de relaciones primarias, intentando no cosificar los bienes relacionales y no olvidar lo que tienen de social y culturalmente construidos; intentando subrayar lo que tienen y deben tener las relaciones familiares $\mathrm{y}$, en general, comunitarias de relaciones libres, elegidas, renovadas y reinventadas a partir de los vínculos de compromiso o sangre; sin olvidar el lado oscuro - los males relacionales de los que habla Pierpaolo Donati-que las relaciones primarias, como todo hecho humano, pueden tener $y$ tienen.

Por otro lado, aunque se esté diciendo que esta actividad que llamamos cuidado parece tener algunas características que la hacen especialmente susceptible de ser vista y tratada como bien relacional, es evidente que también puede ser, debe ser y -en cualquier caso- es gestionada, por ejemplo, como bien privado y, por tanto, como actividad económica lucrativa y servicio profesional (actividad lucrativa y servicio profesional que, por definición, no tienen cabida en el marco de las relaciones primarias). Por otra parte, también es evidente que el cuidado profesional acontece, por ejemplo, cuando el cuidado es entendido y tratado como bien público y se establece, por ejemplo, el derecho a recibir determinados cuidados en determinadas circunstancias. Lo que sí se debe retener, entonces, es que, aunque existe y debe existir cuidado profesional $-\mathrm{y}$ que el cuidado profesional es, legítima y propiamente, cuidado-, 
el cuidado profesional no es funcionalmente equivalente a todos los efectos al cuidado primario.

Así pues, si se admite que el cuidado tiene características que invitan o inducen a su tratamiento, en primera instancia, como bien relacional y si admitimos que no hay equivalencia funcional entre los bienes proporcionados 0 gestionados por los diferentes tipos de agentes, se habrá de asumir que - siendo necesarios tanto el cuidado primario como el cuidado profesionalcada uno de ellos aporta valores - al menos en parte- diferentes. Existe cuidado profesional porque, a todas luces, existen muchos contextos, circunstancias y momentos en los que no existe suficiente cuidado primario idóneo y disponible. Sin embargo, se sigue afirmando - aquí- la preferencia, en primera instancia, por el cuidado primario (del mismo modo que habrá otras actividades o bienes para los que se afirmar en primera instancia la preferencia por una provisión, producción, gestión o protección pública, privada o solidaria). Margarita León recuerda al respecto que "el supuesto, a menudo implícito, de que hay un efecto de sustitución entre el cuidado formal proporcionado por el Estado y el mercado y el cuidado informal dado por la familia no se sostiene empíricamente. En otras palabras, la disponibilidad de servicios de larga duración no desplaza el apoyo familiar. La evidencia más fuerte es proporcionada por Dinamarca, donde el apoyo familiar es alto pero más 'especializado' en menos tareas que demandan tiempo y habilidades” (2014:326).

\section{4. ¿Pueden considerarse los cuidados profesionales como una prestación característica de alguno de los sectores de actividad pertenecientes al ámbito de la acción pro bienestar?}

Para responder a esta pregunta es necesario, también, hacer referencia a un esquema conceptual planteado anteriormente (véase, por ejemplo, Fantova, 2014: 118-124). Según este esquema, en nuestro entorno, el desarrollo de las políticas públicas -y específicamente el de las llamadas políticas sociales - ha determinado que algunos sectores de actividad sean considerados especialmente indicados o propicios para una intervención del Estado que permita garantizar como derecho una cierta satisfacción de una serie de necesidades o, dicho de otro modo, la promoción y protección -tendencialmente universal- de una serie de bienes (que, en esa medida, serían considerados como bienes públicos). Al conjunto de sectores de actividad cubiertos por la política social podemos denominarlo acción pro bienestar en tanto en cuanto, convencionalmente, denominamos bienestar al bien que protege y promueve el conjunto de la política social. En el Cuadro 2 se propone un canon sobre cuáles serían dichos sectores de actividad y cuál sería en cada caso el bien que se protege o promueve y que permite - a su vez-identificar y diferenciar esos distintos sectores de actividad.

\begin{tabular}{|l|l|}
\hline \multicolumn{2}{|l|}{ Cuadro 2. Pilares del bienestar } \\
\hline $\begin{array}{l}\text { Sector de actividad (y } \\
\text { correspondiente política } \\
\text { social y sistema público de } \\
\text { referencia) }\end{array}$ & Bien que protege y promueve \\
\hline Sanidad & Salud \\
\hline Educación & Aprendizaje \\
\hline Servicios sociales & $\begin{array}{l}\text { Interacción (autonomía } \\
\text { funcional/integración } \\
\text { relacional) }\end{array}$ \\
\hline Laboral & Empleo \\
\hline Vivienda & Alojamiento \\
\hline Garantía de ingresos & Subsistencia \\
\hline
\end{tabular}

Fuente: Elaboración propia.

Según el esquema, lo que permite hablar de sectores de actividad en este contexto es la existencia de un bien específico de suficiente importancia como para que pueda ser apreciado (universalmente) por toda la población y, simultáneamente, la existencia de un entramado diferenciado de actividades y estructuras reconocible por parte de dicha población para la respuesta a la necesidad correspondiente (o las necesidades correspondientes) en cada caso. Por ello se entiende que en cada sector de actividad habrá algunas prestaciones, apoyos o actividades características, como lo pueden ser, por citar algunos ejemplos al azar, la operación de apendicitis en la sanidad, la clase sobre la tabla del siete en educación o la reforma de un baño en el ámbito de la vivienda. Igualmente, hay conocimiento de referencia para dichas actividades, conocimiento que nutre las correspondientes cadenas de valor o secuencias de actividades - lógicamente, profesionales- valiosas características de cada sector.

Hay que aclarar que, especialmente en lo tocante a los servicios sociales (social care), la propuesta (presentada más extensamente en Fantova, 2008 o 2014: 207-231) es tentativa y carece de consenso en la comunidad de conocimiento correspondiente. Lo que se quiere decir en la propuesta es que cuando los servicios sociales se hacen cargo de un niño maltratado por sus progenitores, cuando proporcionan ayuda en el domicilio a una mujer mayor en situación de fragilidad que vive sola, cuando potencian los vínculos de apoyo mutuo en los vecindarios o cuando acompañan en su integración comunitaria a un joven con discapacidad en situación de vulnerabilidad, están -en todos los ejemplos- protegiendo y promoviendo la interacción de las personas, entendida como una situación compleja con dos dimensiones: autonomía funcional (capacidad interdependiente para el desenvolvimiento cotidiano) e integración relacional (soporte recíproco y vinculación activa familiar y, en general, comunitaria). 
Se sostiene que la que aquí se denomina interacción es un estado o situación deseable y valiosa, dinámica y cambiante, importante y compleja, como lo son los estados o situaciones de salud, aprendizaje, empleo, alojamiento y subsistencia. Prevenir el deterioro de tales situaciones o estados, ayudar a las personas a alcanzarlos y paliar las consecuencias de su pérdida total o parcial es la labor de las diferentes ramas de la acción pro bienestar. Y se sostiene que, si cada uno de los otros sectores asume su responsabilidad universal sobre el bien que le corresponde, carece de sentido una pretendida intervención residual de los servicios sociales sobre dichos objetos (como alojamiento, empleo o subsistencia), quedando o emergiendo como objeto propio y específico para los servicios sociales ese estado deseable de (relativa) autonomía funcional e integración relacional que se propone denominar interacción.

En cada sector de actividad es crítica la realización del correspondiente diagnóstico o evaluación y de la prescripción o planificación de intervenciones basadas en el conocimiento, entre otras cosas para identificar en qué medida o en qué aspectos corresponde actuar a unos u otros sectores de actividad. Una persona que presenta problemas para conservar su empleo puede requerir, más bien, intervención sanitaria; del mismo modo que puede ocurrir que una que se presenta como necesidad de atención sanitaria deba, sin embargo, ser abordada desde la intervención en el ámbito laboral de la persona.

Sobre esta base, puede afirmarse que el cuidado profesional (o asistencia personal) -tal como viene siendo definido en este artículo- podría ser legítimamente considerado como una prestación, apoyo o actividad propia o característica del ámbito sectorial de los servicios sociales. Ello sería así porque del diagnóstico social que, en los servicios sociales, permitiría caracterizar la situación de interacción de una persona (su capacidad de desenvolvimiento autónomo y el soporte familiar y comunitario idóneo disponible) puede derivarse naturalmente, con mucha frecuencia, la prescripción, planificación o propuesta de cuidados profesionales que complementen el autocuidado y el cuidado primario o comunitario.

Hay que señalar que, en el caso de los cuidados, no sería la complejidad de la actividad o la necesidad de conocimiento más sofisticado la que justificaría la intervención profesional, pues el cuidado profesional retiene la misma característica del cuidado primario, esto es, que -en principio- puede ser realizado, en general, por personas no profesionales. Lo cual no quiere decir que el cuidado - sea o no profesional- no pueda ser perfeccionado mediante la práctica, la formación u otras formas de gestión del conocimiento.

Lógicamente, para que la intervención social - denominando así la actividad operativa que se realiza en los servicios sociales- pueda cumplir su función de protección y promoción de la interacción será necesario que, además -en su caso- de los cuidados profesionales, la persona usuaria $o$ destinataria se beneficie de otras prestaciones, apoyos o actividades más complejas y basadas en conocimiento más sofisticado como la evaluación de los efectos de la intervención o actividades dirigidas a desencadenar cambios en los comportamientos de las personas o en las dinámicas familiares. Así, desde un punto de vista cualitativo, los cuidados profesionales no van a constituir, como tales, el elemento principal de los procesos de prestación de servicios sociales, por más que, desde el punto de vista cuantitativo, puedan constituir una parte importante del paquete de atención. Dicho de otra manera, la prestación de servicios sociales puede conllevar cuidados profesionales pero, necesariamente, los contendrá como parte de un proceso más complejo basado en un conocimiento o cualificación más sofisticada (y científica) de suerte que los cuidados profesionales se brinden de modo que tengan los mayores y mejores efectos que sea posible en la interacción de la persona.

Obviamente una persona puede comprar cuidados profesionales - sin más- en el mercado, del mismo modo que puede automedicarse con determinados fármacos o adquirir un "curso" de inglés en una librería. La diferencia es que, en el caso de la rama sanitaria o la educativa, comparando con la de servicios sociales, existe - al menos en nuestro entorno- una mayor conciencia de la necesidad y el valor del diagnóstico, prescripción e intervención profesionales sobre la base del conocimiento, compatibles - por cierto- con el ejercicio de la autonomía moral y consiguiente capacidad de decisión, elección o consentimiento informado por parte de las personas usuarias. En el caso de los servicios sociales dicha conciencia es menor y posiblemente ello esté relacionado en cierta medida - precisamente- con la valoración social que se da a los cuidados y a las personas que cuidan.

Lo que se quiere decir aquí, en todo caso, es que la decisión sobre los cuidados profesionales que debe recibir una persona pertenece -como la decisión sobre si debe operarse de apendicitis o aprender la tabla del siete - al ámbito de su autonomía moral y capacidad de decisión (o la de quien le represente legítimamente). Pero dicha decisión -como las otras evocadas - pueden y - bajo ciertas premisas y en determinados contextos- deben ser objeto de prescripción facultativa sobre la base de un diagnóstico profesional basado en el conocimiento que permita establecer los mejores ajustes entre cuidado profesional, autocuidado y cuidado primario $y$, en definitiva, alcanzar la mejor interacción y bienestar.

Lógicamente el que se considere el cuidado profesional (o asistencia personal) como prestación propia y característica de los servicios sociales (y a éstos como el marco más adecuado para la prescripción facultativa de cuidados profesionales) 
no excluye que éstos puedan ser proporcionados en el marco de servicios de otros sectores de actividad, del mismo modo que en un hospital (servicio sanitario) se proporcionan prestaciones propias de otros ámbitos, como el alojamiento. Por otra parte, la organización social de los cuidados -y la interacción-, como cualquiera de los grandes bienes de los que se ocupan cada uno de los diferentes sectores de actividad, requiere del concurso -en clave intersectorial o transversal- del resto de sectores: la política laboral, la de garantía de ingresos o la de vivienda - por citar tres- tienen una importancia crítica a la hora de facilitar o dificultar, en cada caso- el equilibrio indicado y deseado entre autocuidado, cuidado primario y cuidado profesional.

\section{5. ¿De qué hablamos cuando hablamos de crisis de los cuidados?}

Se propone denominar crisis de los cuidados a una de las facetas o dimensiones fundamentales de la crisis sistémica o cambio de época que se estaría viviendo en nuestras sociedades en el paso del siglo $X X$ al siglo XXI. Tiene que ver con la confluencia de dos pares de fenómenos. Por un lado la transición demográfica relacionada con el aumento de la esperanza de vida y el envejecimiento de la población en todo el mundo (que, al menos de momento, está suponiendo un aumento de las situaciones de enfermedad crónica y limitación funcional). Por otro lado la progresiva superación de las formas tradicionales de división sexual del trabajo en un contexto de reconfiguración de los tamaños, estructuras, dinámicas, valores y modalidades familiares y convivenciales, con la consiguiente disminución importante de la disponibilidad familiar $y$, en general comunitaria para el cuidado (al menos a día de hoy). En palabras de Cristina Carrasco, “el envejecimiento demográfico junto a la cada vez mayor participación laboral de las mujeres ha llevado a una situación crítica, puesto que la oferta de trabajo de las mujeres no era infinita, como parece que se presuponía. Una situación crítica en relación al cuidado de las personas, básicamente, de las personas mayores muy dependientes que requieren presencia constante de otra persona adulta. Dicha situación, unida a la incapacidad o no disposición para pensar en un cambio de modelo de trabajo ha llevado a la denominada crisis de los cuidados. 'Crisis' que las clases medias y altas han resuelto de manera individual contratando mujeres pobres de países más pobres" (2013: 46).

Amaia Pérez Orozco señala que "la crisis de los cuidados en los países del centro se engarza con la crisis de reproducción social en los países del Sur global que impele a tantas mujeres a migrar. Entre ambas, se conforman las llamadas cadenas globales de cuidados. Con este concepto nos referimos a las redes transnacionales que se establecen para sostener cotidianamente la vida y a lo largo de las cuales los hogares y, en ellos, las mujeres, se transfieren cuidados de unas a otras con base en ejes de jerarquización social" (2014: 214). Yayo Herrero afirma que "si la ignorancia de los límites biofísicos del planeta ha conducido a la profunda crisis ecológica que afrontamos, los cambios en la organización de los tiempos que aseguraban la atención a las necesidades humanas y la reproducción social también han provocado lo que desde algunos sectores del feminismo se ha denominado crisis de los cuidados" (2011: 44). Se nos ayuda, por parte del feminismo, a captar la importancia de la crisis de los cuidados y su interrelación con otras crisis que afectan a la propia sostenibilidad de la vida.

Ciertamente, han sido pensadoras feministas quienes han denunciado frecuentemente el carácter machista y patriarcal de muchas políticas sociales o del Estado de bienestar realmente existente, en la medida en que se basa en una división sexual del trabajo y que, en muchos momentos, ha dado por descontado que las mujeres se ocuparían asimétricamente, de forma subordinada, gratuita y notablemente invisible, de una serie de labores y, específicamente, cuidados en el seno de la familia y la comunidad. Mary Daly y Jane Lewis han afirmado que "si bien el cuidado siempre fue históricamente importante para ser considerado por la política del Estado de bienestar, los desarrollos contemporáneos lo mueven hasta el verdadero centro de la actividad del Estado de bienestar" (2000: 282).

Nos hallamos, por tanto ante una crisis de los cuidados que debe ser vista como una de las dimensiones fundamentales de la crisis de nuestro sistema de bienestar, de nuestro modelo social y de nuestro modelo de vida. Crisis que revela una deficiente organización social de los cuidados, del bienestar y de la vida y que, evidentemente, reparte de forma desigual e injusta sus costes y sufrimientos: más a personas económicamente pobres, más a mujeres que a hombres, más a menores que a adultas, mas a personas con mayores limitaciones funcionales, más a personas que viven en países empobrecidos que a personas que viven en países centrales en la globalización.

La crisis de los cuidados ha sido identificada y analizada desde o con una preocupación especial por la desigualdad entre mujeres y hombres, desde que en 1995 se refiriera a ella Arlie Russel Hochschild (como se recuerda en León, 2014: 1), aunque el cuerpo teórico feminista sobre los cuidados dataría de los años 80 (Martínez Buján, 2011: 96). Sin embargo son esas mismas autoras feministas las que muestran cómo afecta también de forma diferencial e injusta cuando miramos a otros factores de diversidad o situaciones de desigualdad. La propia Hochschild se ha referido a las cadenas globales de cuidados como un trasplante global de corazón.

El análisis de la crisis de los cuidados contribuye a comprender mejor el alcance sistémico de la crisis que está viviendo nuestro modelo social y nuestra sociedad y las limitaciones estructurales de nuestro 
sistema de bienestar, más allá de los recortes que ha sufrido en la recesión económica que comienza en 2008 ó 2009 (en España, pero también en un contexto más amplio). Nos encontramos, en buena medida, ante el reto de reformular el contrato social entre capital y trabajo, pero también entre mujeres y hombres, entre generaciones, entre regiones del mundo, entre las personas que vivimos ahora y las que vivirán en el futuro y en definitiva entre todas las personas en su diversidad de características, situaciones y opciones. El análisis de las economistas y otras feministas sobre la crisis de los cuidados ayuda a colocar la sostenibilidad (global) de la vida (buena) en el centro del diagnóstico y las propuestas. También contribuye a la identificación de valores sociales destructores de la vida y de la naturaleza ética (Goikoetxea, 2014: 60) y humanizadora de la tarea de poner el cuidado de la vulnerabilidad humana en el centro de la vida social, económica y política.

\section{6. ¿En qué medida y en qué sentido son o pueden ser los servicios sociales un elemento clave en la organización social de los cuidados en España?}

Si bien hoy en día en España la mayoría de las personas identifica al Estado como un elemento central o instrumento de referencia cuando se plantea la escolarización de niñas y niños, la atención sanitaria o las pensiones de jubilación, no ocurre, claramente, lo mismo cuando piensa en la organización de los cuidados. Las políticas públicas no han eclosionado en el ámbito de los servicios sociales y de la organización social de los cuidados como lo han hecho en otros ámbitos sectoriales de la acción pro bienestar y en relación con otras necesidades sociales.

La elaboración, aprobación (en 2006), desarrollo y aplicación de la Ley de Promoción de la Autonomía Personal y Atención a las Personas en Situación de Dependencia Ley (39/2006), constituye un interesante experimento natural con el que contamos en España a la hora de evaluar diseños, procesos y efectos en materia de organización social de los cuidados (y más específicamente de los llamados cuidados de larga duración) en los últimos años, máxime cuando la Ley (que contó con un amplio consenso), en su exposición de motivos, se presenta justamente como el instrumento que pretende superar el sistema tradicional de atención a la dependencia en el que el cuidado era asumido por las familias (y fundamentalmente por las mujeres) y dar carta de naturaleza a los servicios sociales públicos españoles - organizados en términos generales como sistemas autonómicos de servicios sociales- como el cuarto pilar que se agregaría a los tres evocados al comienzo de este apartado, con un papel fundamental, precisamente, en lo que tiene que ver con los cuidados de larga duración para las personas en situación de dependencia funcional.
Cabe remitirse a los trabajos de la Asociación Estatal de Directoras y Gerentes en Servicios Sociales (2015, entre otros) para comprobar la influencia de los recortes en el alcance y los recursos en relación con unas previsiones iniciales que también eran limitadas a juicio de muchos análisis. Por dar una única referencia en relación con gasto social, cabe señalar que España dedica a cuidados de larga duración "solo el o,6\% del PIB, mientras Holanda dedica el 3,8\% y Suecia el 3,7\%" (Pazos y Medialdea, 2015: 5).

Sin embargo, sin negar que una apuesta mayor y más sostenida de inversión económica y aportación de recursos públicos es imprescindible para esa eclosión universalizadora de los servicios sociales de la que se hablaba, lo que interesa aquí es apuntar otra hipótesis e identificar otro factor que también haya podido contribuir o esté contribuyendo a que ese cuarto pilar no haya sido levantado con el alcance y los efectos planteados o deseados. Al respecto cabe recordar que, en su artículo 14, la Ley señala que “el beneficiario podrá, excepcionalmente, recibir una prestación económica para el cuidado no profesional". Sin embargo, esa prestación es, con diferencia, la más utilizada en los años que la Ley lleva aplicándose. En la actualidad, según los datos de la web del Sistema de Autonomía y Atención a la Dependencia, en junio de 2105 , la recibe el $38,27 \%$ de las personas beneficiarias. Entre los servicios el más utilizado en el marco de esta Ley es el residencial (15,38\%), debiendo notarse que se trata de un servicio en el que, junto a las prestaciones propias de los servicios sociales, es relevante la de alojamiento (correspondiente, propiamente, a otro ámbito sectorial, según el esquema de referencia). Por ello, junto con otras, sin duda, ha de contemplarse aquí la hipótesis de que un insuficiente perfeccionamiento y posicionamiento de la intervención social y los cuidados profesionales realmente existentes ha influido en esa insuficiente eclosión.

Sea como fuere, la incentivación económica del cuidado primario (o, dicho de otro modo, la compensación económica por el cuidado primario) ha tenido, seguramente, un plus de recorrido porque se contaba en España con las últimas generaciones de mujeres poco incorporadas al mercado de trabajo y altamente disponibles para el cuidado primario y porque el desarrollo y aplicación de la Ley sobre autonomía y dependencia ha coincidido con un brutal repunte de la tasa de desempleo, que en 2010 ya rebasaba la cota del $20 \%$ y en 2012 sobrepasaba el $25 \%$.

En cualquier caso, el proceso de desarrollo y aplicación de la Ley se encontró - aunque con gran desigualdad entre las diferentes comunidades autónomas- con la red preexistente de servicios sociales que, si bien -como se decía- no han eclosionado como cuarto pilar universal, están teniendo un papel insoslayable en el actual modelo español de organización social de los cuidados. En comunidades autónomas más avanzadas en esta materia, como la vasca, se estima que la envergadura de la inversión social pública en el ámbito de los 
servicios sociales equivale ya aproximadamente a un $40 \%$ de la dedicada a sanidad. Según los datos del estudio sobre el gasto público en servicios sociales del Gobierno Vasco, las instituciones vascas destinaron en 2012 una cantidad de 1.636 millones de euros a la financiación de los servicios sociales.

Por tanto, en un deseable contexto de superación de la inequidad de género en el reparto del empleo y del cuidado primario y en una senda necesaria de creación de empleo, parece evidente que los servicios sociales y las políticas públicas de servicios sociales están llamadas a desempeñar un papel más central en la organización social de los cuidados, a la búsqueda del equilibrio idóneo y deseado en cada caso entre autocuidado, cuidado primario y cuidado profesional y del mejor estado de interacción de la persona. La entidad de las necesidades en el área de la interacción y, específicamente, de los cuidados parecen reclamar una gran política sectorial y un sistema público capaz de organizar esa dimensión de la vida social, al menos con tanta razón de ser y tanta capacidad instalada como la que existe en educación, sanidad y pensiones o como la que debiera impulsarse en estos (y otros) ámbitos sectoriales.

Por dar algunas referencias, cabe decir que destaca especialmente la precariedad de nuestra capacidad social agregada y organizada en el ámbito de los cuidados si comparamos, por ejemplo, la cobertura pública y gratuita que tiene en nuestro país la costosa respuesta a determinadas necesidades sanitarias complejas con la ausencia de provisión pública a necesidades de cuidado altamente imbricadas con dichas necesidades sanitarias y que interactúan con ellas. Destaca que las pensiones de jubilación estén permitiendo un considerable flujo de solidaridad descendente (Martínez Virto, 2014: 129-130) en muchas familias (y que la tasa de riesgo de pobreza económica de las personas mayores sea la mitad que la media en España) mientras que las personas mayores pensionistas carecen de un sistema que les brinde seguridad y les facilite hacer previsiones en la organización de sus cuidados actuales o futuros. Destaca que en España, según el censo de censo de 2011 del Instituto Nacional de Estadística en España haya 3.443.365 viviendas vacías y 3.681 .565 viviendas secundarias (es decir, de nuevo, recursos y capacidades infrautilizadas).

Por otro lado es chocante en España el grado de incomparecencia de los servicios sociales en lo que tiene que ver con los cuidados en los primeros años de la vida de la mayoría de la población (salvando, obviamente, los casos de riesgo y desamparo). Siendo evidente que la necesidad que desencadena la búsqueda de servicios profesionales para esas criaturas no es el aprendizaje (objeto de la educación) sino la interacción (objeto de los servicios sociales) y siendo que dichas criaturas necesitan en buena medida cuidados profesionales y no tanto actividades escolares, se ha extendido la idea de que la escolarización es la mejor - o incluso la única- alternativa y que la escuela es la institución a la que corresponde brindar esos cuidados. Sin embargo, basta cruzar la frontera con Francia para conocer alternativas creíbles -y muy utilizadas- a la escolarización en los primeros años, en las que las criaturas son cuidadas por asistentes profesionales en el domicilio y la comunidad, en pequeños grupos flexibles amigables con las dinámicas familiares y convivenciales de las criaturas y de quienes les cuidan. Del mismo modo, desde el ámbito latinoamericano se visibilizan y reivindican formas más comunitarias de cuidado, como, por ejemplo, la de las madres comunitarias de Colombia (Vega y Gutiérrez, 2014: 15).

Parece claro que la organización social de los cuidados es una de las principales amenazas para nuestra sostenibilidad social y que la estrategia de transformación del modelo de cuidados necesita de los servicios sociales como pieza clave. Configurar una política pública en cualquiera de los ámbitos de la acción pro bienestar $-y$, desde luego, en el de los servicios sociales- va mucho más allá de redistribuir dinero para incentivar comportamientos o financiar la compra de servicios en el mercado; representa apostar por construir unos bienes como bienes públicos y utilizar la capacidad del Estado para organizar un ámbito de actividad y la respuesta colectiva, equitativa, inteligente y sostenible a unas determinadas necesidades.

\section{7. ¿Cuáles serían las -0 al menos algunas- condiciones de posibilidad para una contribución más significativa de los servicios sociales a la crisis de los cuidados (considerando el debate en el Reino Unido?}

Acercándonos a la recta final de este artículo, se identifican a continuación tres ejes estratégicos que pudieran resultar inspiradores y eficaces para informar la necesaria apuesta de política pública e inversión social en los servicios sociales como herramienta imprescindible para la respuesta a la crisis de los cuidados y, en definitiva, para la transformación, en claves de calidad, equidad, eficiencia y sostenibilidad de la organización social de los cuidados. Su desarrollo se basará en trabajos anteriores y, para su ilustración en este artículo, se tomarán -como contraste- aportaciones procedentes del debate actual al respecto en el Reino Unido, especialmente interesante por algunas similitudes institucionales (como el papel referencial que siempre ha tenido el National Health Service británico, entre otros instrumentos de la política social) y en todo caso por el vigor y calidad de dicho debate y de sus agentes e instancias de referencia.

Ciertamente uno de los principales factores impulsores o elementos orientadores (drivers) de este debate es el de la preocupación por la legitimación, eficiencia, sinergia y sostenibilidad de la inversión social de los poderes públicos en el sistema de 
bienestar. John Appleby, por ejemplo, se centra en los servicios sanitarios y sociales y afirma que "si los próximos 50 años siguen la trayectoria de los pasados 50 el Reino Unido podría estar gastando una quinta parte de su riqueza en la provisión pública de servicios sanitarios y sociales [...]. Gastar cerca de un quinto del total del PIB del Reino Unido en servicios sanitarios y sociales en los próximos 50 años podría ser asumible -y permitiría un aumento del gasto real en todas las otras áreas de la economía- si las proyecciones para un aumento real del PIB se cumplieran. Claramente, no sería el caso si el crecimiento fuera más lento. Sin embargo, en igualdad de condiciones, ese gasto consumiría alrededor de la mitad de los ingresos del Gobierno y, a pesar de permitir un incremento del nivel real de gasto, significaría reducir la proporción del gasto público en el resto de áreas de alrededor de un $80 \%$ en 2016 a un $50 \%$ para 2061. Por otro lado, a medida que aumenta el gasto, los retornos tienden a disminuir y, a partir de cierto punto, el coste adicional podría llegar a exceder el beneficio en salud, o los beneficios que se podrían obtener gastando en otras áreas diferentes de sanidad y servicios sociales. Todo esto implica que hay importantes decisiones políticas y sociales a tomar acerca de cuanto gastar, en qué gastar y en cómo financiar ese gasto. Esas decisiones no son fáciles e inevitablemente implicarán contrapartidas" (2013: IX-X).

El trabajo de John Appleby, en todo caso, identifica amplísimas discrepancias entre diferentes proyecciones de cara al futuro del gasto relacionado con servicios sanitarios y sociales, así como la diferente naturaleza y sentido de los diferentes elementos tractores de los diversos gastos y subraya el carácter político de las decisiones a tomar, muy relacionadas con los equilibrios y contrapartidas (trade-offs) entre diferentes sujetos sociales. Parece evidente que una mera agenda de incremento de la inversión social pública - sin duda necesariabasada en una mayor recaudación impositiva no necesariamente va a ser apoyada por parte de la ciudadanía ni va a resultar fácil de sostener establemente por parte de los Gobiernos, al menos en el actual marco de la globalización económica. Cabe suponer que la posibilidad de obtener y dedicar -en mayor medida y de la mejor manera- los recursos públicos necesarios a la organización social de los cuidados va a depender en buena medida del acierto e impulso de las tres estrategias que se sugieren a continuación: la primera más vinculada a los procesos operativos de intervención social y, en general, de la acción pro bienestar; la segunda más relacionada con el diseño y organización de las políticas sociales y el sistema de bienestar; y la tercera más relacionada con la gobernanza política y gestión participativa de la protección e inclusión social.
Cuadro 3. Ejes estratégicos de los servicios sociales

1. Innovación tecnológica en una intervención social cada vez más basada en la evidencia y el conocimiento.

2. Los servicios sociales como pieza clave para rediseños y recalibraciones de los sistemas públicos de bienestar desde nuevas visiones de la división e integración intersectorial.

3. Innovación social y política en la participación de los diferentes agentes en el mix de bienestar y el contrato social

Fuente: Elaboración propia.

\subsection{Innovación tecnológica en una intervención social cada vez más basada en la evidencia y el conocimiento}

Hay razones para pensar que el desarrollo y la mejora de los procesos de intervención social (y, en su seno, de los cuidados profesionales) sobre la base del conocimiento y de las preferencias de las personas es una de las estrategias clave para una transformación de los servicios sociales que redunde en un cambio significativo de nuestro modelo de cuidados en consonancia con esa necesidad de perfeccionamiento y posicionamiento de la intervención social y los servicios sociales de la que se hablaba antes.

La intervención social está llamada a seguir el camino de la medicina basada en la evidencia y apoyarse cada vez más en el conocimiento riguroso y en la evaluación de los programas. Para ello es crítica la unificación de las fragmentadas comunidades científicas y de práctica vinculadas a diversas áreas de conocimiento y colectivos poblacionales, cuya dispersión convierte en estériles muchos de los aprendizajes y desarrollos. La consideración del cuidado profesional (asistencia personal) dentro del marco metodológico, técnico y tecnológico de una intervención social centrada en la persona y de enfoque preventivo, participativo, poblacional y comunitario debe permitir superar las miradas excesivamente lastradas por unas $u$ otras perspectivas disciplinares o incentivadas espuriamente para subrayar las reales o pretendidas diferencias entre colectivos y subcolectivos que han ido construyendo determinadas visiones y prácticas - cada vez más obsoletas-de intervención social.

Baste como ejemplo la sorpresa y admiración con la que se presentan experiencias de relación entre las criaturas de una guardería y las personas mayores de una residencia, sin reparar en que es en buena medida la propia visión y organización de la intervención la que ha procedido - previamente- a invisibilizar y destruir las relaciones intergeneracionales que de forma natural se dan en la comunidad, construyendo esos supuestos colectivos objeto de atención, para después descubrir lo positiva que resulta la relación entre los miembros de ambos colectivos.

Nick Andrews et al. subrayan la importancia de la reflexión crítica a partir de la evaluación de la 
intervención y la entrega accesible de resultados y conocimiento para el desarrollo profesional en un sector en el que la relación interpersonal es tan crítica como el de los servicios sociales y en el que el conocimiento práctico, las competencias comunicacionales (más o menos genéricas), el aprendizaje organizacional (en parte implícito), los aspectos éticos y la atención a la voz de las personas usuarias y las cuidadoras primarias es crítica (Andrews et al., 2015: 6-7).

Como en la medicina basada en la evidencia, son fundamentales las revisiones sistemáticas. Así, por ejemplo, en una revisión sistemática de estudios de coste-efectividad sobre atención comunitaria y atención centrada en la persona "se han detectado cinco tipos de intervenciones - la gestión de casos, las ayudas tecnológicas y para la adaptación de la vivienda, los programas de autocuidado, las fórmulas de alojamiento en la comunidad y la atención intermedia- que: a) han sido sometidas a un número elevado de evaluaciones, y b) resultan mayoritariamente coste efectivas [...]. Existe otro grupo de intervenciones - los servicios sociosanitarios integrados, la ayuda a domicilio, y los programas de individualización de la atención- que también resultan mayoritariamente coste efectivos, si bien el número de evaluaciones revisadas es sustancialmente menor, y la base para sostener su coste efectividad es por tanto más limitada" (SIIS Centro de Documentación y Estudios, 2011: 179).

Nigel Keohane, por su parte, refiriéndose a la atención integrada (sanitaria y de servicios sociales) y personalizada (o centrada en la persona) y a las tecnologías de la información y la comunicación, afirma que "las oportunidades para la innovación son tentadoras. La revolución de los datos puede romper las divisiones profesionales, y revolucionar la experiencia del cuidado que tienen las personas, empoderándolas como consumidoras y coproductoras" (2015: 9).

Las tecnologías de la información y la comunicación y otras tecnologías facilitadoras de la vida cotidiana (englobadas, por ejemplo, con el concepto de ambient assisted living o vida cotidiana asistida por el entorno) están introduciéndose de forma prometedora en el ámbito de los cuidados y, en general, de los servicios sociales, potenciando las oportunidades de interacción segura, inteligente, humanizadora y satisfactoria entre las personas y los entornos físicos y humanos de referencia y preferencia para ellas. Especialmente prometedores resultan los desarrollos de las tecnologías de la información y la comunicación, la robótica y la inteligencia ambiental como generadoras del ambiente idóneo para la sinergia entre cuidado primario y profesional (presenciales o no presenciales) y autocuidado en un contexto comunitario.

Resulta evidente, en todo caso, que la dinámica de investigación científica, desarrollo tecnológico e innovación operativa interdisciplinar y personalizadora aquí planteada necesita de una política pública audaz que la impulse, pues la pura tracción del juego de la oferta y la demanda del mercado resulta insuficiente en este momento (como ha ocurrido y sigue ocurriendo, por cierto, en otros sectores de actividad). Dicha apuesta de política pública puede resultar atractiva no sólo por los beneficios que va a reportar el desarrollo de los servicios sociales a sus personas destinatarias y a otros agentes directamente implicados en ellos, sino también por las sinergias de este desarrollo con otros sectores de actividad de alto valor añadido. Por otra parte, seguramente, las innovaciones metodológicas, técnicas y tecnológicas que contribuyen a la organización de los cuidados y a la intervención social surgen y se desarrollan en o desde otros ámbitos sectoriales.

La organización social de los cuidados, para satisfacción de las personas que necesitamos cuidados y de las personas que brindamos cuidados primarios en un marco de dignidad humana, equidad de género y sostenibilidad económica tiene mucho que esperar del desarrollo metodológico, técnico y tecnológico de unos servicios sociales capaces de brindar cuidados profesionales de calidad en un marco de promoción y protección de la interacción.

\subsection{Los servicios sociales como pieza clave para rediseños y recalibraciones de los sistemas públicos de bienestar desde nuevas visiones de la división e integración intersectorial}

En consonancia con la propuesta teórica que se ha hecho y que concibe las grandes políticas sociales sectoriales como entramados de servicios especializados funcionalmente en la respuesta a un área de necesidad (o, dicho de otro modo, en la protección y promoción de un bien) -en principiopara la totalidad de la población, la estrategia de desarrollo de los servicios sociales puede entenderse como una oportunidad en o para procesos de reordenación o reorganización de (y de las relaciones entre) los sectores de actividad o sistemas públicos de servicios. En ese contexto viene recibiendo especial atención la cuestión de la interrelación entre servicios sociales y servicios sanitarios y se ha llegado a decir que "la integración de los servicios sanitarios y sociales se está convirtiendo en el santo grial de la formulación de políticas públicas" (Keohane, 2015: 6).

Estas relaciones intersectoriales han sido estudiadas, por ejemplo, en el informe $A$ New Settlement for Health and Social Care, aprobado por la Comisión Independiente sobre el Futuro de la Atención Sanitaria y Social en Inglaterra (Barker et al., 2014). El informe pone de manifiesto hasta qué punto el desequilibrio entre la inversión en servicios sociales y en sanidad (en una proporción que puede ser de una a siete) genera, por ejemplo, un sistema público de bienestar incapaz de proporcionar ligeros cuidados o apoyos domiciliarios preventivos (desde los servicios sociales) que, sin embargo, incurre 
posteriormente en altos gastos hospitalarios, farmacéuticos o tecnológicos, en buena medida inducidos por la ausencia de esa intervención social más temprana.

El informe permite concluir a The King's Fund que cabría diseñar y mantener de forma equilibrada y coordinada los servicios sanitarios y los servicios sociales con un gasto público incluso inferior al gasto sanitario existente en algunos países. Así, Chris Ham (jefe ejecutivo de The King's Fund) señala en el prólogo del informe que "cuando esté totalmente implementado, el nuevo modelo supondrá alrededor de un $11-12 \%$ del PIB, una cantidad fácilmente comparable con el gasto corriente sólo en sanidad en algunos otros países [...]. Las consecuencias de no hacer nada son que menos personas recibirán servicios sociales financiados públicamente en la medida en que se hacen más recortes en los presupuestos de las autoridades locales y que más organizaciones sanitarias públicas se muestran incapaces de proporcionar acceso oportuno a estándares aceptables de atención en el marco del presupuesto. Todavía más importante, el premio descrito por la comisión -igual soporte para igual necesidad, con los derechos a la atención sanitaria y los servicios sociales más estrechamente alineados entre sí- seguirá siendo un sueño lejano. Las personas con necesidad de cuidado serán forzadas a continuar navegando las complejidades e inconsistencias de los actuales sistemas fragmentados de financiación y derechos [...]. Los costes del cuidado recaerán cada vez más en los individuos y las familias, creando preocupación, incertidumbre e inequidad en una escala que sería inaceptable si se aplicara en la atención sanitaria” (Barker et al., 2014: vi-vii).

En cualquier caso, el informe huye de alimentar determinadas expectativas cuando afirma que los presupuestos para servicios sanitarios y sociales "debieran incuestionablemente ser mayores, con independencia del potencial para ahorros que surja de un sistema más integrado. Esto es en parte porque la evidencia de ahorros financieros por la integración es de momento escasa" (ibídem: ix). Sea como fuere, los argumentos para apostar por un mayor equilibrio, coordinación e integración entre los servicios sociales y los servicios sanitarios son poderosos - por el incremento de la cantidad y complejidad de situaciones en las que las personas presentan entreveradas importantes necesidades correspondientes a ambos sectores- aunque el avance es lento y pocas veces pasa de experiencias piloto en determinadas parcelas de actividad (por ejemplo en atención paliativa), en determinados itinerarios (por ejemplo los de personas con enfermedad mental) o en determinadas zonas (por ejemplo con equipos en los que se comparte el diagnóstico, la gestión de la información o la gestión de los casos). Si bien las tecnologías avanzadas de la información y la comunicación han sido identificadas como un apoyo clave para la coordinación e integración de la atención, la gestión o las estructuras de ambos sectores, hoy por hoy, la asimetría - en términos de conocimiento, envergadura, estructuración o financiaciónentre los dos sistemas aparece como el principal obstáculo.

Por ello, la respuesta a la crisis de los cuidados y la organización social de los cuidados pasaría por repensar y rediseñar conjuntamente las divisiones y relaciones entre, especialmente, tres de las políticas sociales sectoriales mencionadas, apostando por:

- Una transformación de la sanidad que le permita hacer frente mejor al reto de la cronicidad y la haga menos vulnerable a los procesos (o elementos tractores) de la excesiva o inadecuada medicalización, tecnificación y corporativización (que han sido identificados como los principales propulsores del gasto sanitario público por expertos como José Ramón Repullo).

- Un desarrollo del sistema público de servicios sociales que lo configure como pilar de envergadura y reconocimiento equiparable al que tienen la educación o la sanidad, centrado en la interacción (autonomía funcional e integración relacional) de las personas y liberado en términos generales de responsabilidades o intervenciones (relacionadas por ejemplo con la subsistencia o el alojamiento) correspondientes a otros ámbitos.

- Una reorientación de la política de vivienda que confiera más peso a la rehabilitación, la accesibilidad, el alquiler y los espacios y servicios y procesos comunes y compartidos y que la convierta en una verdadera política social orientada a dar respuesta a las necesidades de alojamiento de las personas, especialmente aquellas que, por una u otra razón, presentan una mayor vulnerabilidad (y necesidad de apoyo) residencial.

La organización social de los cuidados que reclama la crisis de los cuidados y el necesario fortalecimiento del sector de los servicios sociales puede apoyarse en la recalibración (desinversiones en unas áreas e inversiones en otras áreas) de la inversión social pública, orientándola estratégicamente para apoyar rediseños inteligentes y nuevas dinámicas sectoriales e intersectoriales que permitan al sistema de bienestar adaptarse proactivamente a los nuevos riesgos sociales, con especial atención a la interfaz (y, por ejemplo, a la interoperabilidad de sistemas de información) ente sanidad y servicios sociales, especialmente sensible para las personas con mayor necesidad de cuidados.

\subsection{Innovación social y política en la participación de los diferentes agentes en el mix de bienestar y el contrato social}

Del mismo modo que -como se acaba de decir- el desarrollo de los servicios sociales necesita apoyarse en (e impulsar) una revisión de las divisiones y 
relaciones intersectoriales y una recalibración de las diferentes políticas sectoriales dentro del sistema de bienestar, puede dar (y beneficiarse de) la oportunidad de experimentar, en clave de innovación social y política, fórmulas más participativas, flexibles, dinámicas y sinérgicas de relación entre los diferentes agentes implicados. Así, por ejemplo, se ha identificado la importancia de las fórmulas e instrumentos de contratación y concertación de servicios como apoyo e impulsor para la atención integrada (Addicott, 2014).

En este terreno revisten especial interés las experiencias y modelos que buscan el empoderamiento de la persona que recibe los cuidados. Es el caso, por ejemplo, de los pagos directos (direct payments) o presupuestos individuales (individual budgets) para la asistencia personal y otros servicios que la administración pública entrega a la persona usuaria para que ésta decida libremente su uso y, particularmente, el proveedor o los proveedores de productos o servicios (eventualmente con el asesoramiento o ayuda de una persona gestora de caso, que actuaría como bróker). A más complejidad del caso, es decir, a más número y envergadura de actividades y agentes involucrados, más necesidad de que se haga una gestión del caso, esto es, más necesidad de que alguien asuma algún tipo de responsabilidad sobre el conjunto de la intervención y coordine esa multiplicidad de agentes y actividades involucradas en el proceso de intervención. De hecho, en el ámbito anglosajón, se observa un desarrollo paralelo de la gestión de caso (case management) con la implantación de modelos de gestión de la atención (care management) caracterizados por la división de papeles entre quien financia, quien prescribe, quien elige y quien presta los servicios. Suelen apoyar con fuerza estos modelos los movimientos de autodefensa, autorrepresentación, autodeterminación y autogestión de diferentes categorías o grupos de personas con necesidad de cuidados profesionales (Zalakain, 2013: 207).

Según Natasha Curry y Chris Ham, “la evidencia procedente de los servicios sociales en el Reino Unido sugiere que los pagos directos han llevado a una mayor satisfacción de las personas usuarias, mayor continuidad de la atención, menos necesidades insatisfechas y un uso más costeefectivo de los recursos públicos [...]. A pesar de esta evidencia, la utilización sigue siendo baja [...]. La baja utilización puede ser explicada por la manera en la que los pagos se han operativizado, más que porque el concepto en sí esté viciado. La evaluación de los presupuestos individuales en servicios sociales mostró algunos resultados positivos, aunque parecían variar entre diferentes grupos poblacionales" (2010: 38-39).

En todo caso, como señalan Catherine Foot et al., estos programas no son sino una de las opciones -ni la única ni obligatoria - a la hora de respetar y promover el mayor control de las personas que necesitan cuidados (y, en su caso, de sus cuidadores primarios) sobre su propia vida, siendo necesario aceptar y potenciar la capacidad de decisión y participación activa de las personas en todos los pasos y momentos de su itinerario de atención: diagnóstico, prescripción, planificación, prestación del servicio, gestión, financiación, seguimiento, evaluación e investigación (Foot et al., 2014).

Rick Muir e Imogen Parker vinculan la necesidad de implicación de las personas usuarias con la complejidad creciente a la que han de hacer frente los servicios y políticas públicas y afirman que "necesitamos reconfigurar radicalmente nuestros servicios públicos para que sean más capaces de afrontar los complejos riesgos que tenemos delante. La reforma de la administración pública ha confiado demasiado en el uso de herramientas burocráticas o basadas en el mercado que están mal equipadas para enfrentar problemas con un creciente grado de complejidad, desde la enfermedad crónica al desempleo de larga duración” (2014: 1). Según esta aportación del Institute for Public Policy Research "el cambio hacia un Estado más relacional debe tener lugar en dos niveles. Primero, en el nivel de los sistemas públicos de servicios, hay una necesidad de mayor conectividad. Esto requiere la descentralización de los presupuestos de servicios al nivel local; la integración de presupuestos y, a veces, de las organizaciones; mayor autonomía para la primera línea combinada con transparencia acerca de los resultados; y establecimiento de infraestructuras colaborativas para que los actores y las instituciones puedan aprender y mejorar. En segundo lugar, en el nivel micro, necesitamos fomentar relaciones profundas, tanto entre los ciudadanos como entre usuarias y profesionales. En la práctica esto puede tomar la forma de profesionales líderes que asumen la responsabilidad de casos individuales, mayor trabajo de base vecinal y la creación proactiva de instituciones que reúnen a las personas usuarias y aumentan su capacidad de ayudarse" (ibídem, 2014: 74).

Las propuestas para una mayor implicación de las personas que reciben cuidados en el marco de los servicios de los que son usuarias son congruentes con la aplicación del enfoque comunitario o la atención comunitaria (community care) que apuesta por fortalecer - dentro de los sistemas de servicios- la capacidad resolutiva del nivel de atención más general y próximo a las personas, su domicilio, sus redes y entornos. En el caso español esta apuesta se hizo con notable éxito en el caso de la sanidad pero está en buena medida por hacer en el caso de los servicios sociales. Según Public Health England, “usando la metodología del retorno social de la inversión (SROI), una metodología específica para evaluar el valor, un análisis del desarrollo comunitario en autoridades locales reportó un retorno de 2,6 libras por cada libra invertida" (2015: 34).

A su vez estas experiencias de participación de usuarias y desarrollo comunitario están vinculadas con la promoción del voluntariado y el tercer sector. 
Chris Naylor et al. reconocen y reivindican el valor añadido por las personas (más de tres millones en la sanidad y servicios sociales del Reino Unido) y organizaciones voluntarias y llaman la atención sobre la diversificación e innovación que se está produciendo en el ámbito de la acción voluntaria, por ejemplo con los bancos de tiempo. Señalan, sin embargo, que los contextos de recortes y privatizaciones amenazan con desnaturalizar las iniciativas solidarias y que una falta de visión estratégica puede llevar a las personas y organizaciones a roles confusos, pérdida de confianza y tensiones entre agentes. Apuestan por no utilizar la acción voluntaria para reducir costes sino para aumentar la calidad relacional de los servicios, con un impacto que debe ser medido (2013: vii-viii).

El marco de la Big Society, propuesto por David Cameron en 2010 impulsa -y a la vez sesga - estos debates en torno a experiencias de innovación social y política para abrir el juego a nuevas relaciones entre agentes. Sin embargo el marco privatizador no es el único para dichas experiencias y debates que, en muchas ocasiones, pueden orientarse precisamente para un perfeccionamiento, transformación y fortalecimiento de las políticas y sistemas públicos, especialmente necesario en el sector de los servicios sociales. Nos encontramos, en todo caso, ante el debate sobre la función del Estado y, en definitiva, el contrato social que regula lo que se espera de la ciudadanía y lo que ésta puede esperar de los poderes públicos.

\section{Cuatro metáforas para terminar}

Mientras se escribía este artículo, cuatro metáforas emergían para expresar lo que aquí se ha desarrollado. Según la primera de ellas, en lo que tiene que ver con la organización social de los cuidados nos encontraríamos, como en el dilema del prisionero, perdiendo todas por no cooperar, por no considerar los cuidados en mayor medida como bienes relacionales, públicos y comunes. Según la segunda metáfora, esta situación, como bomba de racimo, perjudica a muchas y muy diversas personas $y$, por tanto, reúne condiciones para convertirse en un exitoso tema (issue) de agenda política con el marco (frame) e impulso adecuados. Sin embargo, en ese contexto podemos -especialmente los sectores mejor instalados en el actual sistemaactuar como Sansón, agarrándonos sin más a los pilares o columnas (del sistema de bienestar) más tradicionales y reconocidos - vinculados a los viejos riesgos sociales - (con el resultado de que podamos hacer caer el edificio) o podemos apostar-arriesgando política, profesional y ciudadanamente- por levantar un nuevo pilar - los servicios sociales como oferta creíblea la vez que transformamos el modelo de bienestar y el contrato social, para la sostenibilidad de la vida, para el buen vivir (sumak kawsay, en quechua) en el que-ecológicamente- nos reconocemos como seres diversos y vulnerables -iguales en dignidad y derechos - que necesitamos cuidar y ser cuidados. 


\section{Bibliografía}

ADDICOTT, R. (2014): Commissioning and Contracting for Integrated Care, Londres, The King's Fund.

\section{ASOCIACIÓN ESTATAL DE DIRECTORAS Y GERENTES EN} SERVICIOS SOCIALES (2015): XV Dictamen del Observatorio de la Ley 39/2006 de Promoción de la Autonomía Personal y Atención a las Personas en Situación de Dependencia, Madrid.

ALIENA, R. (2012): "Regímenes de bienestar y política social por otros medios: un marco analítico", Comunitania. Revista Internacional de Trabajo Social y Ciencias Sociales, nํ 4, págs. 9-42.

ANDREWS, N. et al. (2015): Developing Evidence-enriched Practice in Health and Social Care with Older People, York, Joseph Rowntree Foundation.

APPLEBY, J. (2013): Spending on Health and Social Care over the Next 50 Years. Why Think Long Term?, Londres, The King's Fund.

BARKER, K. et al. (2014): A New Settlement for Health and Social Care, Londres, The King's Fund.

CARRASCO, C. (2015): "El cuidado como bien relacional: hacia posibles indicadores", Papeles de Relaciones Ecosociales y Cambio Global, nํㅜ 128, págs. 49-60.

- (2013): “El cuidado como eje vertebrador de una nueva economía", Cuadernos de Relaciones Laborales, vol. 31, n- 1, págs. 39-56.

CASADO, D.; y SANZ, M. J. (2012): Crianza saludable. Fundamentos y propuestas prácticas, Madrid, Siposo.

CHARLESWORTH, A.; y THORLBY, R. (2012): Reforming Social Care: Options for Funding, Londres, Nuffield Trust.

CURRY, N.; y HAM, C. (2010): Clinical and Service Integration. The Route to Improved Outcomes, Londres, The King's Fund.
DALY, M. (2002): “Care as a good for social policy”, Journal of Social Policy, vol. 31, ํㅜㄹ, págs. 251-270.

DALY, M.; y LEWIS, J. (2000): "The concept of social care and the analysis of contemporary welfare states", British Journal of Sociology, vol. 51, no․ 2, págs. 281-298.

DICKINSON H. et al. (2012): The Role of Third Sector in Delivering Social Care, Londres, School for Social Care Research.

EDWARDS, N. (2014): Community Services. How They Can Transform Care, Londres, The King's Fund.

ESPAÑA (2006): “Ley 39/2006, de 14 de diciembre, de Promoción de la Autonomía Personal y Atención a las Personas en Situación de Dependencia", Boletín Oficial del Estado, no 299, 15-12-06, págs. 44.142-44.156 [rhttps://www.boe.es/ boe/dias/2006/12/15/pdfs/A44142-44156. pdf>].

FANTOVA, F. (2014): Diseño de políticas sociales. Fundamentos, estructura y propuestas, Madrid, CCS.

- (2010): “Actividades básicas de la vida diaria”, en DE LUCAS, F.; y ARIAS, A. (dir.): Diccionario internacional de trabajo social y servicios sociales, Buenos Aires, Miño y Dávila, pág. 19.

- (2008): Sistemas públicos de servicios sociales. Nuevos derechos, nuevas respuestas, Bilbao, Universidad de Deusto.

FOOT, C. et al. (2014): People in Control of Their Own Health and Care. The State of Involvement, Londres, The King's Fundación; National Voices.

GOIKOETXEA, M. (2014): "La ética como referente de la atención integral y centrada en la persona”, en RODRÍGUEZ RODRÍGUEZ, P.; y VILÀ, A. (coord.): 
Modelo de atención integral y centrada en la persona. Teoría y práctica en ámbitos del envejecimiento y la discapacidad, Madrid, Tecnos, págs. 59-83.

GUÉRIN, S. (2010): De l'État providence a l'État accompagnant, París, Michalon.

HAM, C.; y WALSH, N. (2013): Making Integrated Care Happen at Scale and Pace, Londres, The King's Fund.

HERRERO, Y. (2011): "Propuestas ecofeministas para un sistema cargado de deudas", Revista de Economía Crítica, nํ13, págs. 30-54.

HM GOVERNMENT (2012): Caring for our Future. Reforming Care and Support, Londres.

JARAíZ, G.; y GONZÁLEZ PORTILLO, A. (2014): "Transformaciones y retos relacionales en el ámbito de los servicios sociales comunitarios", Documentación Social, $\mathrm{n}$ ำ175, págs. 133-156

KEOHANE, N. (ed.) (2015): A Problem Shared. Essays on the Integration of Health and Social Care, Londres, Social Market Foundation.

LEICHSENRING, K. et al. (2013): Long-term Care in Europe. Improving Policy and Practice, Londres, Palgrave Macmillan.

LEÓN, M. (ed.) (2014): The Transformation of Care in European Societies, Londres, Palgrave MacMillan.

MARTÍNEZ BUJÁN, R. (2011): “La reorganización de los cuidados familiares en un contexto de migración internacional”, Cuadernos de Relaciones Laborales, vol. 21, nํㅜ 1 , págs. 93-123.

MARTÍNEZ VIRTO, L. (2014): “Una crisis interminable: estrategias para resistir y primeros síntomas de sobrecarga en las familias", Zerbitzuan, nํ5ㄱ, págs. 121-136 [<http://www.zerbitzuan. net/documentos/zerbitzuan/Crisis_ interminable_estrategias_resistir_sintomas_ sobrecarga_familias.pdf`].

MUIR, R.; y PARKER, I. (2014): Many to Many. How the Relational State Will Transform Public Services, Londres, IPPR.

NAYLOR, C. et al. (2013): Volunteering in Health and Care. Securing a Sustainable Future, Londres, The King's Fund.

PAZOS, M.; y MEDIALDEA, B. (2015): Reorganizar el sistema de cuidados: condición necesaria para la recuperación económica y el avance democrático, Madrid, Podemos.

PÉREZ OROZCO, A. (2014): Subversión feminista de la economía. Aportes para un debate sobre el conflicto capital-vida, Madrid, Traficantes de Sueños.

PUBLIC HEALTH ENGLAND (2015): A Guide to Communitycentered Approaches for Health and Wellbeing, Londres.

RENDUELES, C. (2013): Sociofobia. El cambio político en la era de la utopía digital, Madrid, Capitan Swing.
SIIS CENTRO DE DOCUMENTACIÓN Y ESTUDIOS (2011): Atención comunitaria y atención centrada en la persona: revisión de estudios de costeefectividad, Donostia-San Sebastián, SIIS Centro de Documentación y Estudios.

TOBío, C. et al. (2010): El cuidado de las personas. Un reto para el siglo XXI, Barcelona, Fundación La Caixa.

TORNS, T. (dir.) (2014): Nuevas profesiones para la organización social del cuidado cotidiano, Barcelona, Universidad Autónoma de Barcelona.

VEGA, C.; y GUTIÉRREZ, E. (2014): “Nuevas aproximaciones a la organización social del cuidado. Debates latinoamericanos", Iconos, ํㅜ50, págs. 9-26.

ZALAKAIN, J. (2013): “Tendencias y prácticas innovadoras en inclusión social. Perspectiva internacional”, Lan Harremanak, no 29, págs. 171-212.

\section{Algunas webs para seguir el debate sobre servicios sociales en el Reino Unido}

Active and Assisted Living Programme: 〈http://www.aal-europe.eu〉.

Community Care: 〈http://www.communitycare.co.uk〉.

Care Quality Commission: 〈http://www.cqc.org.uk〉.

European Social Network: 〈http://www.esn-eu.org〉.

Fòrum d'Innovació i Excel-lència en Serveis Socials i Salut: 〈http://www.forumitesss.com〉.

Health and Social Care Information Centre: 〈http://www.hscic.gov.uk〉.

Joseph Rowntree Foundation: 〈http://www.jrf.org.uk〉.

The King's Fund: 〈http://www.kingsfund.org.uk〉.

National Voices: 〈http://www.nationalvoices.org.uk〉.

Nesta: 〈http://www.nesta.org.uk〉.

National Institute for Health and Care Excellence: 〈http://www.nice.org.uk〉.

Nuffield Trust: 〈http://www.nuffieldtrust.org.uk〉.

Personal Social Services Research Unit: 〈http://www.pssru.ac.uk〉.

Social Care Institute for Excellence: 〈http://www.scie.org.uk〉.

SIIS Centro de Documentación y Estudios: 〈http://www.siis.net〉.

Social Care Network: <http://www.theguardian.com/social-carenetwork〉.

International Journal of Integrated Care: 〈http://www.ijic.org〉. 